\title{
Hospital Readmissions Among the Homeless Population in Albuquerque, New Mexico
}

\author{
Victoria F. Dirmyer* \\ State of New Mexico, NMDOH, Santa Fe, NM, USA
}

\section{Objective}

To analyze a homeless population, demographically and by health condition, over a 3-year time period who were admitted to an Albuquerque area hospital.

\section{Introduction}

It is estimated that there are 1.7 million homeless individuals in the United States; $36 \%$ are families with children under the age of 18 . Due to lack of resources, homeless individuals frequent emergency departments for immediate health care needs. Homeless individuals are hospitalized more often, and once hospitalized have longer lengths of stay and incur higher hospital costs compared to non-homeless individuals ${ }^{1-2}$.

\section{Methods}

This study is a retrospective analysis of hospital inpatient and discharge data from 50 non-federal hospitals across the state of New Mexico. The primary outcome for this analysis was a 30-day readmission for a homeless individual, counted from the date of hospital discharge to the date of the next hospital admission. A homeless record was defined by the patient's address; either recorded as 'homeless', 'none', or an address for a shelter in Albuquerque. Patient records that had at least one instance of being homeless and an admission to an Albuquerque hospital were included in the analysis. Once identified as a homeless patient, all records for that patient were included in the readmission analysis. Records were analyzed from 2010-2012. The comparison group for this analysis consists of homeless individuals who were admitted to Albuquerque area hospitals, but did not have a 30-day readmission during the analysis time period.

\section{Results}

In all three years, males were proportionately higher in number. The overall mean age over the three year time period was 43.8 years. The predominant admitting primary diagnosis for homeless patients was Neuro-Psychiatric conditions, followed by Digestive Diseases and Alcohol and Drug Related conditions. Most readmissions occurred early after discharge, with two-thirds of the readmissions occurring prior to 10 days after discharge $(66.3 \%)$.

Roughly, one third of the homeless patients experienced a 30day readmission as an inpatient during the three year time period. Approximately $45 \%$ of patients had multiple inpatient admissions each year, with some patients being admitted more than 10 times in one calendar year ( $2 \%$ of patients). The average 30 -day readmission rate among Bernalillo County residents (who did not identify as homeless) was $12.3 \%$ over the same time period.

In adjusted analyses, factors significantly associated with an increased odds of a 30-day readmission included age, gender, certain primary diagnoses, and the number of admissions per patient (Table 2).

\section{Conclusions}

In this three year, city centric study, the 30-day inpatient readmission rate among patients who identified as homeless was 30.1 percent. Given the high readmission rate observed in this study and the work conducted by prior researchers with a similar population, hospitals need to take appropriate steps to identify this population as they come through their doors and create a suitable discharge plan of action for this population to prevent costly readmissions.

\begin{tabular}{|c|c|c|c|}
\hline Calendar Year & $\begin{array}{c}\text { Number of } \\
\text { Patients with a 30- } \\
\text { Day Readmission }\end{array}$ & $\begin{array}{l}\text { Total Number of } \\
\text { Patients }\end{array}$ & $\begin{array}{l}\text { \% of Patients with a } \\
\text { 30-Day Readmission }\end{array}$ \\
\hline \multicolumn{4}{|l|}{ Homeless Patients } \\
\hline 2010 & 107 & 368 & 29.1 \\
\hline 2011 & 82 & 253 & 32.4 \\
\hline 2012 & 93 & 317 & 29.3 \\
\hline Overall* & 256 & 850 & 30.1 \\
\hline Number of Records & 720 & 2,068 & 34.8 \\
\hline \multicolumn{4}{|c|}{ Bernalillo County Residents } \\
\hline Overall* & 17,798 & $144,710^{+}$ & 12.3 \\
\hline
\end{tabular}

30-Day Readmission Rates by Year, Bernalillo County, 2010-2012

\begin{tabular}{|c|c|c|}
\hline \multirow{3}{*}{$\begin{array}{l}\text { Patient Characteristics } \\
\text { Gender }\end{array}$} & Unadjusted OR (95\% CI) & Adjusted OR (95\% Cl) \\
\hline & Ref. & Ref. \\
\hline & $0.65(0.46-0.93)^{*}$ & $0.77(0.58-1.03)$ \\
\hline \multicolumn{3}{|l|}{ Age Groups (Years) } \\
\hline $0-19$ & Ref. & Ref. \\
\hline $20-29$ & $3.90(1.18-12.98)^{*}$ & $2.52(0.90-7.07)$ \\
\hline 30-39 & $4.66(1.44-15.07)^{*}$ & $2.89(1.05-7.97)^{*}$ \\
\hline $40-49$ & $4.26(1.33-13.65)^{*}$ & $2.65(0.97-7.28)$ \\
\hline $50-59$ & $4.54(1.42-14.48)^{*}$ & $2.68(0.98-7.35)$ \\
\hline $60+$ & $5.69(1.66-19.58)^{*}$ & $3.23(1.11-9.37)^{*}$ \\
\hline \multicolumn{3}{|l|}{ Race/Ethnicity } \\
\hline White & Ref. & Ref. \\
\hline Black & $0.99(0.54-1.82)$ & $0.94(0.59-1.52)$ \\
\hline Native American & $0.78(0.46-1.33)$ & $0.77(0.52-1.17)$ \\
\hline Hispanic & $0.84(0.58-1.22)$ & $0.79(0.59-1.06)$ \\
\hline Other & $0.60(0.31-1.17)$ & $0.82(0.48-1.40)$ \\
\hline \multicolumn{3}{|l|}{ Hospitalization Characteristics } \\
\hline Number of Admissions & $1.38(1.31-1.43)^{*}$ & $1.37(1.31-1.43)^{*}$ \\
\hline Length of Stay (LOS) & $1.00(0.99-1.00)$ & $1.00(0.99-1.00)$ \\
\hline \multicolumn{3}{|l|}{ Diagnosis Category } \\
\hline Neuro-Psychiatric Condition & Ref. & Ref. \\
\hline 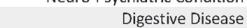 & $0.92(0.59-1.44)$ & $0.89(0.59-1.35)$ \\
\hline Alcohol or Drug Related Disease & $0.72(0.44-1.19)$ & $0.76(0.48-1.20)$ \\
\hline Respiratory Disease & $0.48(0.27-0.84)^{*}$ & $0.49(0.29-0.83)^{*}$ \\
\hline Injury & $0.48(0.25-0.89)^{*}$ & $0.62(0.35-1.10)$ \\
\hline Cardiovascular Disease & $0.48(0.27-0.84)^{*}$ & $0.62(0.37-1.03)$ \\
\hline 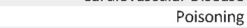 & $0.92(0.52-1.63)$ & $0.75(0.42-1.32)$ \\
\hline Other & $0.98(0.70-1.37)$ & $1.03(0.76-1.40)$ \\
\hline \multicolumn{3}{|l|}{ Discharge Status } \\
\hline Routine Discharge & $0.83(0.64-1.08)$ & $1.02(0.58-1.80)$ \\
\hline Discharge Against Medical Advice & $1.44(0.98-2.13)$ & $1.79(0.93-3.44)$ \\
\hline Discharge to Specialty Facility & $0.98(0.69-1.38)$ & $1.18(0.63-2.22)$ \\
\hline
\end{tabular}

Patient Hospitalization Characteristics and Odds of 30-Day Readmission, Bernalillo County, 2010-2012

\section{Keywords}

Homelessness; Health Inequalities; Readmission; Hospital Stay

\section{Acknowledgments}

The authors would like to thank all NM residents and general hospitals. The Health Systems Epidemiology Program is partially funded by a grant from the National Syndromic Surveillance Program (CDC).

\section{References}

1. Doran KM, Shumway M, Hoff RA, Blackstock OJ, Dilworth SE, Riley ED. Correlates of hospital use in homeless and unstably housed women: The role of physical health and pain. Womens Health Issues. 2014 Sep-Oct;24(5):535-41.

2. Hwang SW, Weaver J, Aubry T, Hoch JS. Hospital costs and length of stay among homeless patients admitted to medical, surgical, and psychiatric services. Med Care. 2011 Apr;49(4):350-4.

\section{*Victoria F. Dirmyer}

E-mail: victoria.dirmyer@state.nm.us 